\title{
DNA Detection Technology Using Zinc Finger Protein
}

\author{
Takenori Kumagai ${ }^{1}$, Koichi Abe ${ }^{1}$, Wataru Yoshida² and Kuzunori Ikebukuro ${ }^{1 *}$
}

${ }^{1}$ Department of Biotechnology and Life Science, Tokyo University of Agriculture and Technology, Tokyo, Japan

${ }^{2}$ School of Biotechnology and Bioscience, Tokyo University of Technology,1404-1 Katakuramachi, Hachioji City, Tokyo, Japan

\begin{abstract}
With recent advances in nanotechnology and sequencing technology, DNA diagnostic technology is becoming practical and is advancing at a fast pace. In these detection systems, mainly PCR (particularly real-time PCR) and DNA probe hybridization techniques are used. We suggest that detecting PCR products using double-stranded DNA (dsDNA) is more convenient and powerful compared with DNA probe hybridization technique. Zinc finger protein is the major DNA binding protein in nature and it recognizes dsDNA with sequence specific manner. Additionally, by changing its amino acids sequence, we can design it to recognize the desired DNA sequence to some extent. Using zinc finger protein for DNA detection element, simple, accurate and sensitive DNA detection can be achieved. In this review, dsDNA detection using zinc finger protein is described and compared with recent advanced technology.
\end{abstract}

Keywords: DNA sequences; Metastatic cancer; Legionella pneumophila; DNA methylation

\section{Introduction}

Detection of specific DNA sequences has become an important technology in health care, bioterrorism prevention, and genotyping. Some systems are commercially available, such as for diagnosis of single-nucleotide polymorphisms (SNPs) [1], metastatic cancer [2], or epigenetic modification [3]. These systems reveal present, congenital, or future disease problems. SNPs are a genotyping method that reveals differences in one individual's genome from another's and can also provide insights into the most effective drug regimen for a particular patient or reveal susceptibility to specific diseases. Given that DNA methylation [3] and histone modification [4,5] play important roles in gene regulation, epigenetics has become the focus of attention for many researchers and clinicians [6].

In clinical diagnosis, genetic information should be obtained not only by determining the presence, absence, or abundance of particular genetic information but also with simplicity, rapidity, and accuracy [7]. Many DNA detection methods have been described, and some are already commercially available. There are few methods that do not require initial treatments, such as PCR or extraction and purification from biological samples using immune chromatography, often used in clinics [8]. These methods are simple and rapid and may be useful in several fields but often give false-positive or false-negative results because of the presence of unrelated DNA or inhibitors or quenchers [9]. Because false results lead to wrong treatment of patients, these methods may cause unintended health hazards or critical illnesses [10]. Given that in the near future, DNA diagnostic systems will be routinely applied to many patients, their diagnostic accuracy will be critically important.

With recent advances in technology, the efficiency and rapidity of DNA amplification methods, such as PCR, are increasing [11]. Chrissy [12] has reported a diagnostic assay for ocular Chlamydia trachomatis infections using digital PCR assay. Microfluidic droplet generator chips are used to partition PCR 1-nl volume micelles that are stable at high temperatures. The droplet PCR is performed in a normal thermal cycler within a few minutes. This technology enables us to perform simple and rapid testing for target DNA amplification.

PCR products must be detected by rapid, simple, and highly sensitive detection methods and with its signal transducer. Electrophoresis is the DNA detection method most routinely used by researchers and is now becoming a more useful technology [13]. Despite its convenience, it detects only the length of DNA and may give false-positive test result. Most currently used detection methods involve DNA probe hybridization, which uses the sequence-specific recognition of single-stranded DNA (ssDNA) [14]. It requires only denaturation of PCR products and annealing with complementary ssDNA to make rapid detection possible. With respect to its sequence specificity, it detects target PCR products accurately. In the last 10 years, several technologies have been proposed and have become commercially available. Nakamura et al. developed a multi-sample detection system employing a tag insertion primer and an electrochemical DNA chip [15]. It is mixed and injected into a cassette that contains the DNA chip with immobilized probes and automatically performs hybridization, washing, and signaling. Sawata has reported a DNA probe hybridization technology using PNA probe technology [16]. PNA is an artificial oligoamide that is capable of forming highly stable complexes with oligonucleotides complementary to DNA sequences because of its neutral electric charge. Real-time PCR is also a useful technology for detecting specific DNA [17]. It detects target DNA by amplification. A fluorescent dye and a quencher-modified DNA probe (e.g., Taqman ${ }^{\circ}$ probe) hybridize to the template DNA during PCR, and DNA polymerase dissociates the DNA probe. The fluorescent dye and the quencher draw apart from each other, generating a fluorescent signal. The fluorescent signal increases relative to DNA amplification, indicating the rapidity and reliability of real-time PCR. Besides this technology, in recent years, a novel technology that uses a sequencespecific direct double-stranded DNA (dsDNA) recognition element has been developed. It does not need denaturation and hybridization, increasing its speed. In the process of DNA probe hybridization, most DNA re-hybridizes to the original ssDNA, reducing signal intensity.

*Corresponding author: Kuzunori Ikebukuro, Department of Biotechnology and Life Science, Tokyo University of Agriculture and Technology, Tokyo, Japan, Tel: 81 42-367-5504; E-mail: kazikebu@gmail.com

Received September 06, 2015; Accepted September 14, 2015; Published September 21, 2015

Citation: Kumagai T, Abe K, Yoshida W, Ikebukuro K (2015) DNA Detection Technology Using Zinc Finger Protein. J Microb Biochem Technol 7: 278-281. doi:10.4172/1948-5948.1000225

Copyright: @ 2015 Kumagai T, et al. This is an open-access article distributed under the terms of the Creative Commons Attribution License, which permits unrestricted use, distribution, and reproduction in any medium, provided the original author and source are credited. 
Because sequence-specific dsDNA detection detects PCR products directly, it permits the rapid and sensitive detection of target PCR products. Osawa et al. have reported a PCR product detection method from Legionella pneumophila using zinc finger protein (ZFP), which is the most common DNA-binding motif [18]. ZFP recognizes DNA in a sequence-specific manner with high affinity $(K d: \mathrm{nM}-\mathrm{pM})$. Moreover, its binding mode is simple, and it is relatively easy to construct an artificial ZFP that binds to a specific DNA sequence $[19,20]$.

Several ways to detect specific DNA sequences have been described above (Figure 1). In this review, we focus on useful DNA diagnostic technology including recent advanced techniques such as a direct dsDNA detection method using sequence-specific DNA recognition elements. Along with these techniques, we will discuss their application and the possibility of their use in DNA diagnostic technology in the future.

\section{dsDNA recognition technology: Sequence-specific DNA- binding protein}

There are many DNA-binding motifs in nature, including leucine zipper and helix-turn-helix [21]. Jarzy described a simple method for isolating specific DNA sequences directly from genomic DNA [22]. They used a fusion protein, the sequence-specific binding protein DnaA fused with glutathione $S$-transferase (GST). DnaA recognizes the oriC coding region, the key region for the initiation of chromosomal DNA replication. Takeuchi et al. have reported the detection of PCR products from the genus Salmonella using DnaA IV, the DNA recognition domain of DnaA protein [23].

Given that naturally occurring DNA-binding proteins recognize specific sequences or repeat sequences like those of EcoRI, it is difficult to distinguish many DNA sequences. Ideally, for DNA diagnosis as described above, any DNA sequence should be distinguishable by its high affinity and specificity to a DNA-binding protein. One candidate is the zinc finger motif, the largest functional group encoded in the eukaryotic genome. TheCys2-His2 type of zinc finger motif is among the most common types of DNA-binding motif, and its individual zinc finger consists of approximately 30 amino acids in a conserved bba configuration. Several amino acids on the surface of the helix typically contact 3-4 bp in the major groove of DNA, with varying levels of selectivity. The modular structure of ZFPs has made them an attractive framework for the design of custom DNA-binding proteins $[20,24,25]$. Key to the application of ZFPs for specific DNA recognition is the development of unnatural arrays that contain three to six tandem zinc finger domains that recognize 9-19 bp [26]. Cliff has described a DNA detection method using ZFP, fusing a half fragment of asignal-producing protein in a process named sequence-enabled reassembly (SEER) detection $[27,28]$. In recognizing a specific DNA sequence, half of the fragments of a protein reassemble while emitting a corresponding signal. GFP and -lactamase has reported fusing one half of the signal protein with a natural zinc finger (zif268) and other half of the signal protein with an artificial reported protein (PBSII). Given its enzymatic activity and low background in colorimetric assays, the b-lactamase system could detect $20 \mathrm{nM}$ of purified target DNA within 5 min. Applying this system to a DNA microarray with immobilized artificial ZFP on poly (ethylene glycol) hydrogel permitted the detection of several DNA samples at the same time [29]. Although SEER is rapid and simple, its sensitivity is not sufficient for DNA detection. The enzymatic activity of a separated protein is decreased at least 10 times compared with that of a full-length enzyme. Kazutoshi et al. have described a method for detection of dsDNA by FRET using GFP and CFP with ZFP [30]. They fused a dimerization peptide to its $\mathrm{N}$ terminus to enhance the dimerization of two distinct ZFPs, achieving a sensitivity of 10-30 nM that considerably exceeded that of SEER-GFP or SEER-LAC.

In the application of DNA detection technology with zinc finger, rapidity and simplicity are important. We attempted to develop a dsDNA detection method using ZFP and not relying on protein reassembly or fluorescence detection.

\section{A PCR-product detection system for pathogenic bacteria using ZFP}

L. pneumophila is a major pathogenic bacterium that is difficult to detect rapidly using the culture method, because the bacteria grow slowly. We have developed a rapid and specific detection method using

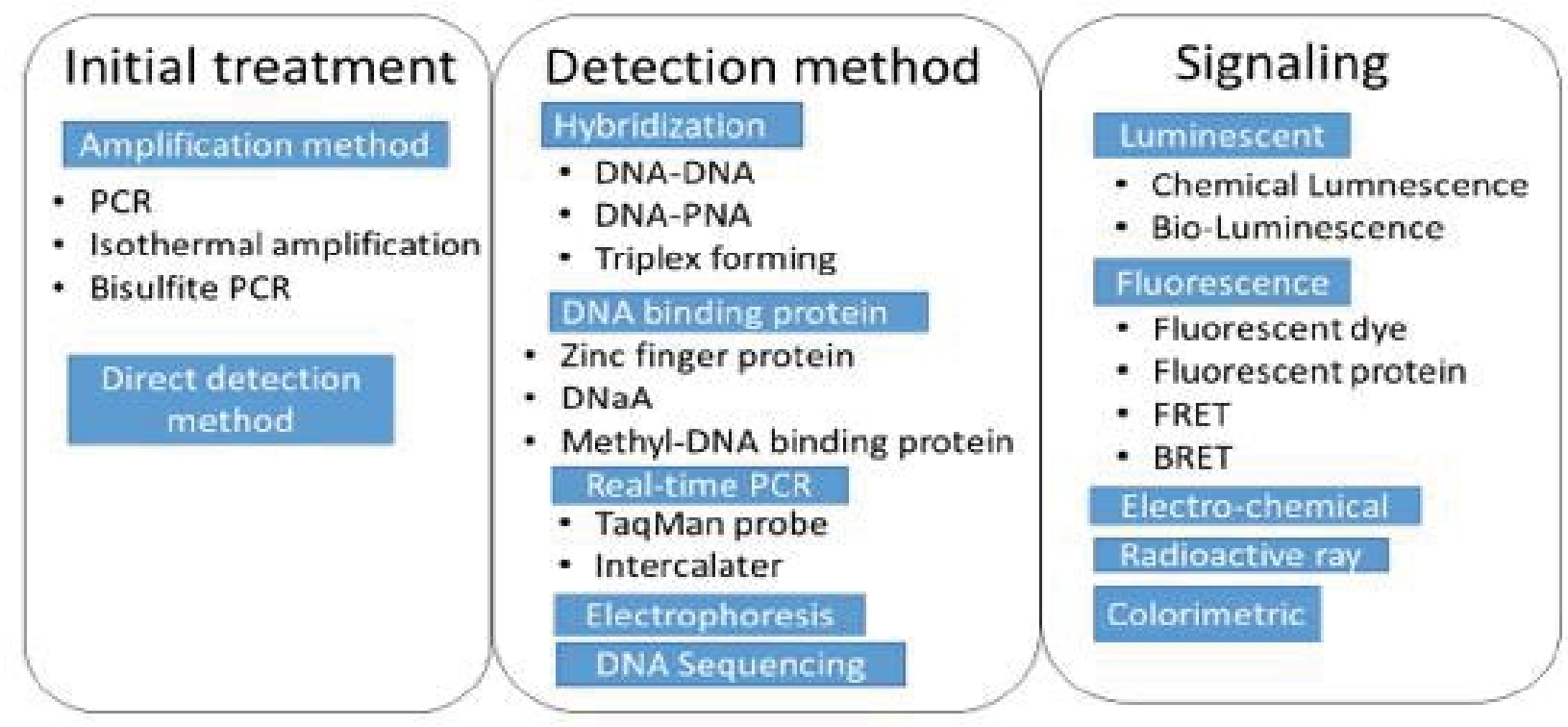

Figure 1: Classification for the strategy for detecting DNA. 
PCR products amplified from a $L$. pneumophila genome DNA using a ZFP (Figure 2) [18]. The PCR target region was a 49-bp sequence of a L. pneumophila specific region containing a 9-bp Sp1-binding site and two 20-bp primer regions at both ends and is located in the flhA gene in the L. pneumophila genome. The PCR product was detected by ELISA using horseradish peroxidase-conjugated anti-GST antibody. Only 100 copies of L. pneumophila genome were detectable by this detection system. Moreover, using fluorescence depolarization measurement, we were able to detect the PCR products from the L. pneumophila genome within only $1 \mathrm{~min}$. Applying this technology to other pathogenic bacteria, Salmonella spp. and the influenza A virus using Zif268 and Sp1, respectively, which is the most researched protein in ZFP [31].

To improve the rapidness and sensitivity of the assay, we constructed a fusion protein of a signal-producing enzyme and ZFP [32]. Of many types of signaling enzyme, we focused:* on firefly luciferase. Luciferase produces a luminescence signal by catalyzing the oxidation of luciferin in the presence of ATP, $\mathrm{Mg}^{2+}$, and molecular oxygen. The excited state of oxyluciferin then returns to the ground state, accompanied by the emission of light with a high quantum yield $(0.88 \pm 025)$. Thus, a highly luminescent signal is observed. Using its substrate specificity against luciferin, it is possible to construct an assay with low background noise [33-35]. Compared with a colorimetric detection or a fluorescent detection assay, a luminescent molecule emits a light signal by its original reaction, leading to a low background signal. These features of luciferase, high signal and low noise (leading to a high $\mathrm{S} / \mathrm{N}$ ratio), are key characters of biosensors. The use of a fusion protein of luciferase and ZFP (LUC-ZF) permitted the detection of $100 \mathrm{fmol}$ of synthetic dsDNA. In comparison with the ELISA described above, the detection limit was improved more than 100 times. We found that 10 copies of the L. pneumophila genome was detectable using PCR and LUC-ZF, but theoretically 35 cycles of PCR from 1 copy of the genome generate approximately $100 \mathrm{fmol}(235 / 6 \times 1023)$ of dsDNA, so that one copy of the bacterial genome may be detectable. Electrochemical detection is also a useful detection method, owing to its high sensitivity and amenability to miniaturization, for example, in glucose sensing [36]. We focused on glucose dehydrogenase $(\mathrm{GDH})$ in place of luciferase as a signal-producing enzyme [37]. For combining an electrochemical detection system with PCR, it is rather easy to construct an on-chip total analysis system to detect pathogenic bacteria. Ten copies of Escherichia coli genome were detectable in this system, the same result as achieved by LUC-ZF.

\section{Detection of DNA and histone modification using ZFP}

Methylation of DNA at cytosine bases is an important mechanism widely used to regulate gene expression and transposable elements in eukaryotic organisms [38]. Aberrant methylation is detected in the promoter regions of tumor suppressor genes in tumors, and this aberrant methylation pattern is dependent on the type of tumor [39]. Thus, aberrant DNA methylation is regarded as a biomarker in the early diagnosis of cancer. The sodium-bisulfite sequencing assay has been widely used in DNA methylation analysis but requires several steps to produce results and is accordingly time consuming. We have developed a rapid and sensitive detection method for methylated DNA using a fusion protein of a methyl CpG-binding domain (MBD) with ZFP and LUC-ZF, w named methylated DNA precipitation combined luciferase-fused

ZFP assay (Figure 3) [40]. In the first step, methylated DNA is captured by MBD from a sonicated human genome and immobilized on glutathione-coated magnetic beads via GST. Next, the eluted DNAs from beds and the target region, which contains a zinc finger recognition site, are amplified by PCR using biotinylated primers. Finally, PCR products are immobilized on streptavidin-coated magnetic beads via biotin and detected by LUC-ZF.

Similarly to DNA methylation, histone modifications in specific target genes are regarded as biomarkers [41]. DNA methylation is used for long-term gene silencing, whereas an immediate gene expression state is determined by histone modifications. Detection of histone modifications in specific target regions is important not only for diagnosis but also for evaluation of drug treatment. We have reported a detection system for histone modifications at target genomic regions by chromatin immune-precipitation combinedLUCZF-based bioluminescence resonance energy transfer (ChIP-ZFBRET) assay (Figure 4) [42,43]. At the first step, ChIP was performed to collect the modified histone. Next, PCR was performed against target genomic region, which includes a zinc finger recognition site. At last, homogeneous detection of the PCR product by BRET was performed by LUC-ZF and fluorescent DNA intercalating dye that is excited at luciferase luminescence. To develop a high-sensitivity detection system, we performed a solid-phase detection assay with a bound/free separation procedure using LUC-ZF. In the histone modification detection assay, we demonstrated the BRET assay, which is performed homogeneously. Compared with a solid-phase detection assay, a homogeneous detection assay is more rapidly and conveniently
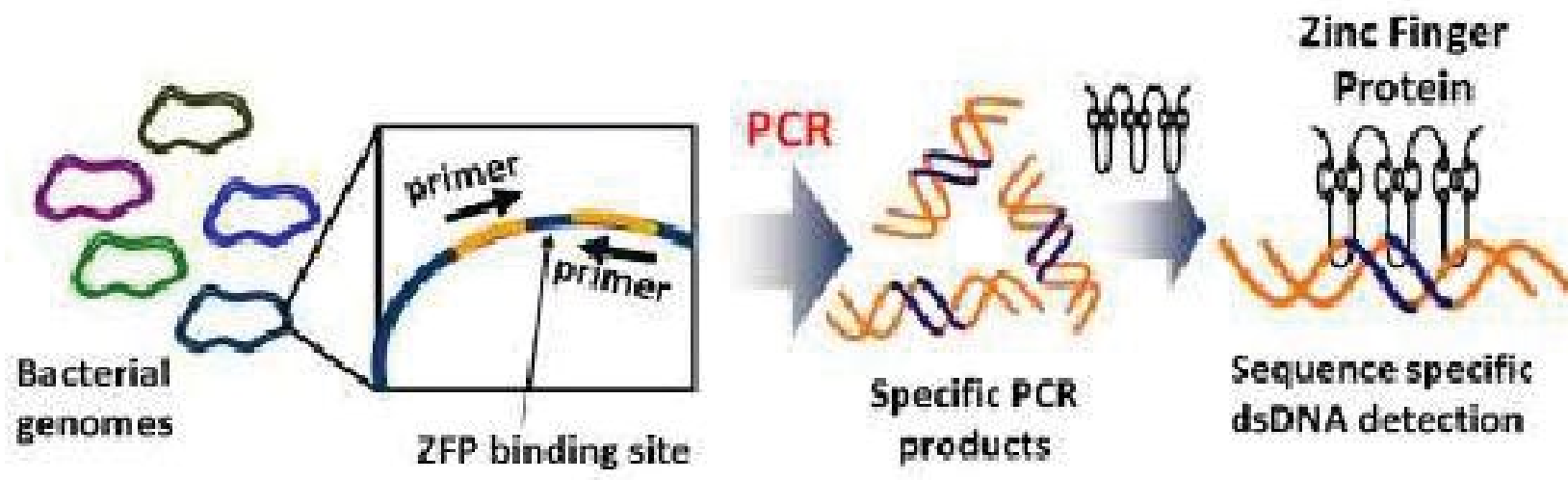

Figure 2: Bacterial genomic DNA detection method using zinc finger protein. A specific region of bacterial genomic DNA that contains the zinc finger protein recognition region was amplified by PCR. The PCR product was detected by zinc finger protein. 


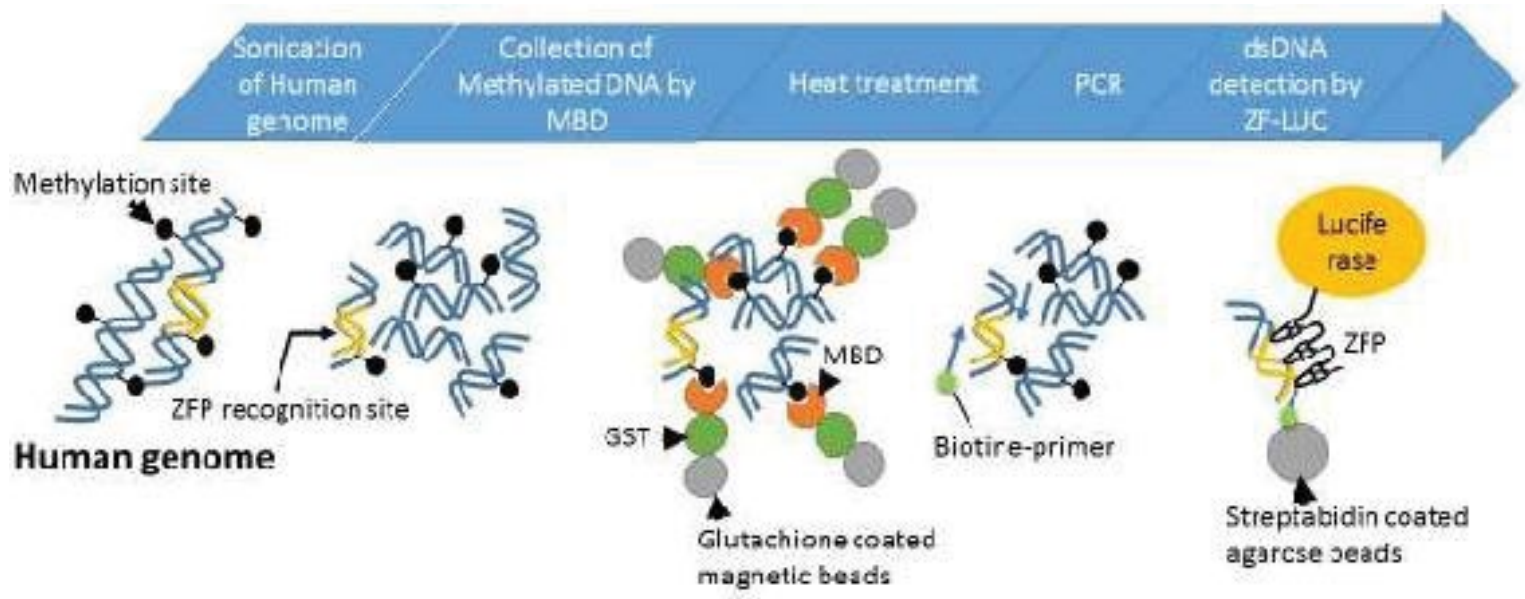

Sonicated digested methylated DNA was collected by Methyl binding domain.

Collected methylated DNA was amplified by PCR and detected by ZF-LUC.

Figure 3: Detection scheme of MELZA.

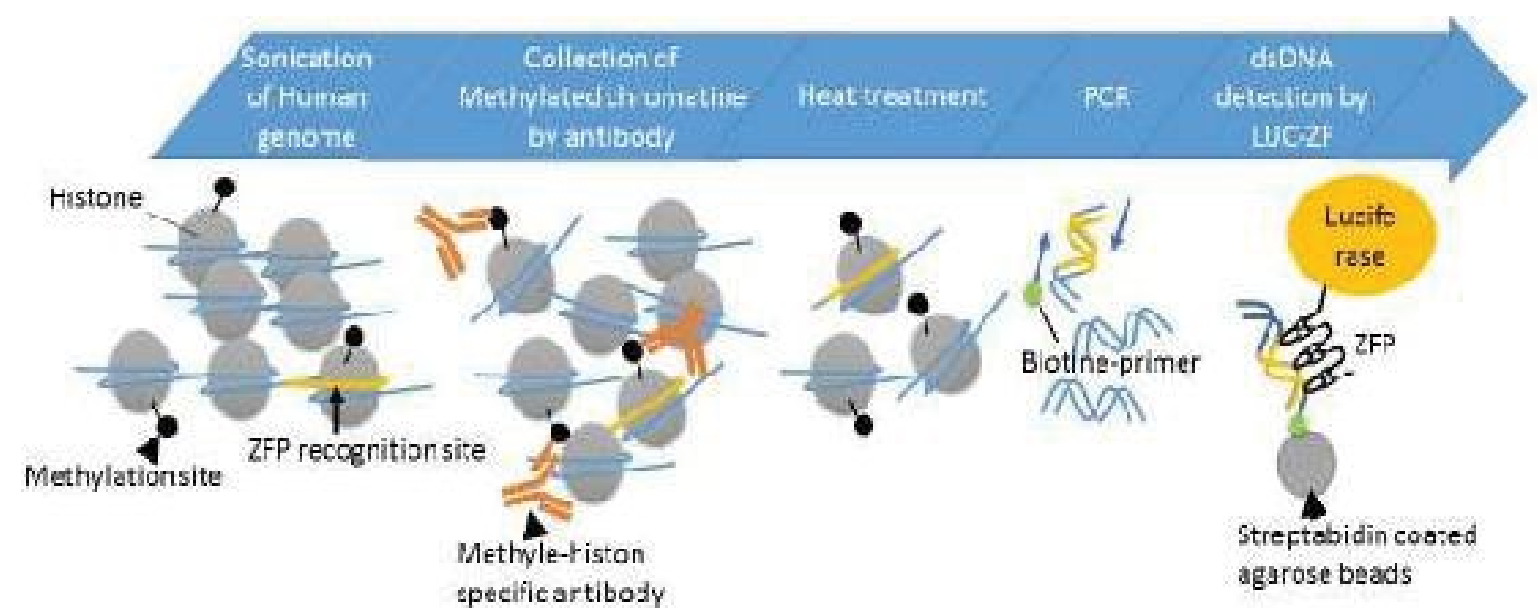

Chromatin immunoprecipitation was performed using target histone modification antibodies. The target region containing a zinc finger recognition site was amplified by PCR. The PCR product was detected by BRET using ZF-luciferase and a fluorescent DNA intercalating dye that is excited by luciferase luminescence.

Figure 4: ChIP-ZF-BRET assay.

performed. A mixture of LUC-ZF and the fluorescent molecule BOBO3 was added to the PCR product from chromatin DNA that contains a zinc finger recognition site, and 550 and $602 \mathrm{~nm}$ emission spectra were measured after the addition of the luciferase substrate. Emission at 602 $\mathrm{nm}$ was not increased in the presence of non-target dsDNA.

\section{Comparison with real-time PCR technology}

Real-time PCR produces a signal in proportion to the amplification of specific DNA. Use of TaqMan probe DNA is a powerful tool for analyzing sequences of PCR products. However, real-time PCR systems using the TaqMan probe can be affected by sample impurities, and the inhibitory activity of impurities against DNA polymerase depends on the type of DNA polymerase [44-48]. This observation suggests that suitable DNA polymerases may not always be used in real-time PCR because the system requires DNA polymerases that exhibit exonuclease activity. Thus, a sequence-specific post-PCR detection system is required for detection of pathogens in real samples.

Another aspect of real-time PCR is that it depends on detecting the amplification of DNA using a fluorescent signal. Although fluorescence detection is a useful method, it requires a special device for signal detection and is thus not preferred to use as an on-site detection tool. Shunsuke et al. have developed a rapid and sensitive method for detection of bacteria using a microchip with real-time PCR [48]. To develop a portable detection system, they detected PCR products by endpoint detection of fluorescent signal. By contrast, the standard PCR method is not limited to fluorescence detection but can detect by luminescence or electrochemically, as described above. In particular, electrochemical detection methods are suitable for compact PCR detection systems [38].

\section{A new candidate for a dsDNA-binding molecule}

Transcription activator-like effectors (TALEs) are transcriptional factors encoded by pathogenic bacteria of the genus Xanthomonas to alter transcription of genes in host plant cells. TALEs are injected into host plant cells via the type III secretion system and bind to genomic 
DNA to alter transcription in these cells, thereby facilitating pathogenic bacterial colonization $[49,50]$. DNA binding by TALE is mediated by highly conserved 33- to 35-amino acid repeat arrays. Each TALE repeat in an array specifies a single base of DNA determined by amino acids at positions 12 and 13 of a domain called repeat variable di-residues (RVDs). Crystal structures of TALE DNA-binding domains bound to their cognate sites reveal that individual repeats comprise a two-helix $\mathrm{V}$-shaped structure and that the amino acids at positions 12 and 13 are positioned in the DNA major groove. By engineering those residues, a simple and useful TALE recognition code was constructed [51]. The authors provided experimental evidence for the TALE repeat code by constructing the first examples of engineered TALE repeat arrays with novel specificities [52]. The TALE repeat code was also confirmed by another group via computational analysis of the binding specificities of the TALE [53]. Subsequent reports provided additional evidence that engineered TALE repeats with desired specificities can be created using the code. At present, nearly all engineered TALE repeat arrays use four domains with the residues NN, NI, HD, and NG, for the recognition of $\mathrm{G}, \mathrm{A}, \mathrm{C}$, and $\mathrm{T}$, respectively. There are several interactions involved in recognition of DNA by TALE, including basic amino acid direct hydrogen bonds, weaker van der Waals interaction, and hydrophobic interactions such as by steric exclusion of interactions with alternative nucleotides. Interestingly, using HD and NG in RVD, unmethylated and methylated cytosines may be discriminated, because NG recognizes a methylated cytosine, whereas HD does not. Moreover, a well-conserved thymine is present at the 0 position of most natural TALE target sites [52,54]. T0 recognition probably serves as a binding anchor from which the protein wraps around a DNA helix and probes a nucleotide sequence $[55,56]$. Too many strong RVDs in the N-terminal part of the TALE DNA interaction domain may recognize a non-target sequence. Thus, owing to its simple recognition of DNA, an artificial TALE is rather easy to construct and may have particular specificity to the target site, but its recognition is asymmetric across the proteinDNA interface, and it binds preferentially to DNA. This observation suggests that an engineered TALE does not always recognize the target DNA with high affinity and specificity.

Several applications using TALE for DNA-binding domains have been reported in the past 5 years. Properties of the TALE DBD offer great potential for research, biotechnology, and gene therapy, such as fusing activation, silence domain [57], or nuclease, which is often called TALEN [58]. TALEN is a fusion protein consisting of a TALE DNA-binding domain with a FokI nuclease domain that is often used in zinc finger nuclease (ZFN) technology [59]. ZFN technology has yielded substantial achievements in a variety of model organisms and cell types [51] that were previously inaccessible by classical gene targeting methods. The technical knowhow that was established in ZFN technology was subsequently adapted to TALEN. TALEN has been successfully used for targeted genome editing in yeasts, plants, and eukaryotes [60-63].

\section{Engineering method for ZFP}

Almost 20 years have passed since an artificial ZFP was reported. To date, many artificial ZFP selection methods have been developed, but at present three construction technologies are mainly used: modular assembly [64], selection-based methods in bacteria $[65,66]$, and a proprietary technology owned by Sangamo BioSciences [67].

The modular assembly generates candidate ZFPs for target sequence against 3 bp by phage display [68] or derived from natural ZFPs [69] and links them into finger modules targeted to a specific sequence. Modular assembled ZFNs are functional in several organisms [70,71] and human cell lines [72] with relatively low toxicity. Against this simplicity, many studies have shown that the success rate for isolating ZFP with high specificity is low. The reason may be the recognition complexity of the zinc finger-DNA interaction. However, Ramirez et al reported that modular assembly had unexpectedly high failure rates, with only $6 \%$ of zinc finger module pairs predicted to produce an active ZFN. Zinc finger recognizes target 3 base by $-1,2,3,6$ positions in a-helix making hydrogen bond contact but each individual finger interact with each other and constructing the structure stability. For example, an aspartate in position 2 of a-helix recognizes mostly $\mathrm{C}$ and sometimes A in the opposite strand of the neighboring DNA triplet [73], thus requiring a $5^{\prime}-\mathrm{GNN}$ or $5^{\prime}-\mathrm{TNN}$ as an adjacent triplet in the target site [74].

Context-dependent selection of zinc finger arrays has been established in vitro [75] and also in bacteria [65,76]. Among them, the most successful selection system is named OPEN and is based on the bacterial two-hybrid assay. The method involves two distinct steps. First, low restricted parallel selections are performed for binding of randomized fingers to each triple finger in the targeted sequence. Next, fingers from these pools are linked by a combinatorial method and the products are selected at high restricted environment for binding to the final target. ZFPs derived from the OPEN system show high activity with low toxicity and have been applied to a few model organisms [65] and human cell lines. Success rates of $25 \%$ have been achieved with ZFN $[77,78]$. However, OPEN is complicated and laborious, with 5-8 weeks needed for identifying a ZFP with high $K d$ and specificity to specified DNA. Also, the library is limited to arrays that recognize all 16 GNN (e.g., GAG, GCT) and a few TNN triplets. An advanced method reported by Sander et al. is Context-Dependent Assembly (CoDA), which is the rapid assembly of parts of OPEN arrays. CoDA ZFNs were shown to successfully cleave $50 \%$ of their chromosomal targets. However, the range of targetable sequences was a subset of OPEN and insufficient to target.

Sangamo BioSciences has developed a proprietary platform based on two specialized fingers of three finger domains with the 9-aa linker -LRQKDGERP- [67]. Applying this approach, they have reported 103-fold greater affinity than three fingers of a two-finger domain. Researchers may purchase customized ZFNs made by the Sangamo approach through the Sigma-Aldrich CompoZr service, but the cost of these proteins limits the scale and scope of projects that can be performed.

Underlying such a study of ZFP, several prediction algorithms for contact between zinc finger and DNA have been developed. These design tools enable us to save labor and time in selecting specific ZFPs. SVM Model [79]; ZIFIBI [80], ZiFiT [81], ZiF-BASE [82], ZiF-Pridict [83], zinc finger tools [84], ZiFDB [85], enoLOGOS [86], and predicting DNA recognition [87] have been developed and are available on Web sites [88].

\section{Comparison with ZFP and TALE}

Owing to its simple recognition mode, TALE is easy to design against new DNA targets [89]. In contrast, it is rather difficult to construct a designated ZFP that recognizes a specific sequence. Indeed, in application to the therapeutic field-like ZFN, it prefers high sequence specificity and selectability of DNA sequence because of off-target effects derived from nonspecific binding or frame shifts. However, for use of these proteins in particular fields, ZFP may function more effectively than TALE. The main reason is the large MW of TALE. The single finger of the zinc finger recognizes three base pairs, in contrast 
to one base pair for each TALE module, meaning that ZFP is more compact than TALE. Focusing on this property, there are three main advantages. First, for constructing a fusion protein with functional protein (e.g., a nuclease, transcriptionally activated protein or labeled enzyme (luciferase, GDH)), several functional protein properties decrease. We have compared firefly luciferase enzymatic activity by fusing only one unit of ZFP with that using two units of zinc finger and GST. Enzyme activity decreased $1 / 5-1 / 10$ in the first fusion protein and $1 / 10-1 / 100$ in the second fusion protein (data not shown). Luciferase consists of two domains, the active center lies between two domains, and when it reacts with luciferine and ATP, two subunits interact with each other and promote the oxy-luciferil reaction. Thus, the mobilities of both proteins are important for reaction, so that fusing a large protein or several units to the luciferase decreases its activity. Second, the expression and purification efficiency of luciferase decrease in proportion to its MW. Third, background signal derived from nonspecific interaction increases with MW in the DNA detection step, because

sometimes the number of amino acids participates in nonspecific interaction with base material. At the present stage, zinc finger prefers to bind GNN triplets, and the GNNGNNGNN recognition site has an occurrence probability of $1.56 \%$. It is difficult to insert specific DNA at specific sites in genome manipulation but in pathogenic bacterial detection method is not so restricted by narrow recognition field. Using bioinformatics tools like BLAST, it is almost possible to confirm whether a target bacterium is detectable. Additionally, recently designed ZFPs are becoming easier to construct with high DNA sequence specificity. Cong et al. has described the selection of two-finger modules recognizing GRNNYG and selecting the nonGNN recognition finger [90]. An estimation strategy of artificial zinc finger that contains a non-GNN triplet has been developed [91-93]. Sangamo Biosciences and Sigma-Aldrichhave started a construction service of customized ZFPs made by the Sangamo approach through the CompoZr service, but the cost approaches $\$ 25,000$ and limits the scale and scope of projects that can be performed [94]. The zinc finger consortium has offered several ZFPs constructed by Joung and Voytas using the OPEN system or CoDA. These zincfinger-coded plasmids are available in Addgene. Also, ZiFiT, the zinc finger consortium, offers online software as an effort to provide a simple and easy tool for ZFP design [80]. It is a popular Web tool that provides an integrated modular design approach by incorporating three different datasets enumerating zinc finger-binding patterns for independent modules developed by Barbas, Sangamo, and ToolGen [95-99]. The user may enter a query DNA sequence and choose one or more of these sets. Scores are given with predictions of their chances of success, as measured by a bacterial two-hybrid assay. Indeed, the range of recognition sequences and the design strategy of zinc finger are expanding.

\section{Conclusion}

With recent advances in DNA diagnostic systems, anyone can read individual genomes or SNPs to understand his or her health status. In parallel, simple, rapid, and accurate DNA detection systems are also becoming more important. DNA-DNA hybridization including realtime PCR is the standard method. DsDNA detection using a DNAbinding protein does not need steps such as denaturation of dsDNA and recognizes in a sequence-specific manner, so that it is a simple, rapid, and accurate detection method. Engineered ZFPs have been studied for almost 20 years and are still expanding in utility. A nucleotide recognition module named TALE is becoming a bioengineering tool at an explosive pace and is gradually taking the place of ZFP. In gene manipulation applications, TALE may be more useful, but its large MW may limit its advancement.

As an alternative to TALE, ZFP is one of the most compact DNA recognition motifs in nature. Advances in technology will determine the better approach for dsDNA detection.

\section{References}

1. Ghosh I, Stains Cl, Ooi AT, Segal DJ (2006) Direct detection of doublestranded DNA: Molecular methods and applications for DNA diagnostics. Mol Biosyst 2: 551-560.

2. Ciombor KK, Berlin J (2014) Targeting metastatic colorectal cancer - present and emerging treatment options. Pharmgenomics Pers Med 7: 137-144.

3. Hernández HG, Tse MY, Pang SC, Arboleda H, Forero DA (2013) Optimizing methodologies for PCR-based DNA methylation analysis. Biotechniques 55: 181-197.

4. KarliÄ $\ddagger$ R, Chung HR, Lasserre J, Vlahovicek K, Vingron M (2010) Histone modification levels are predictive for gene expression. Proc Natl Acad Sci U S A 107: 2926-2931.

5. Bachman KE, Park BH, Rhee I, Rajagopalan H, Herman JG, et al. (2003) Histone modifications and silencing prior to DNA methylation of a tumor suppressor gene. Cancer Cell 3: 89-95.

6. Duffy MJ, Napieralski R, Martens JW, Span PN, Spyratos F, et al. (2009) Methylated genes as new cancer biomarkers. Eur J Cancer 45: 335-346.

7. Lazcka O, Del Campo FJ, Muñoz FX (2007) Pathogen detection: A perspective of traditional methods and biosensors. Biosens Bioelectron 22: 1205-1217.

8. Bergamini S, Bellei E, Reggiani Bonetti L, Monari E, Cuoghi A, et al. (2014) Inflammation: An important parameter in the search of prostate cancer biomarkers. Proteome Sci 12: 32.

9. Mano J, Hatano S, Futo S, Minegishi Y, Ninomiya K, et al. (2014) Development of direct real-time PCR system applicable to a wide range of foods and agricultural products. Shokuhin Eiseigaku Zasshi 55: 25-33

10. Gonçalves-de-Albuquerque Sda C, Pessoa E Silva R, de Morais RC, TrajanoSilva LA, Régis-da-Silva CG, et al. (2014) Tracking false-negative results in molecular diagnosis: proposal of a triplex-PCR based method for leishmaniasis diagnosis. J Venom Anim Toxins Incl Trop Dis 20: 16.

11. Renvoisé A, Brossier F, Sougakoff W, Jarlier V, Aubry A (2013) Broad-range PCR: Past, present, or future of bacteriology? Med Mal Infect 43: 322-330.

12. Roberts CH, Last A, Molina-Gonzalez S, Cassama E, Butcher R, et al (2013) Development and evaluation of a next-generation digital PCR diagnostic assay for ocular Chlamydia trachomatis infections. J Clin Microbiol 51: 2195-2203.

13. Southern EM (1975) Detection of specific sequences among DNA fragments separated by gel electrophoresis. 98: 503-508.

14. Gooding J (2002) Electrochemical DNA Hybridization Biosensors Electroanalysis 14: 1149-1156.

15. Nakamura N, Ito K, Hashimoto M, Nakamura A, Hayashimoto N, et al. (2011) Development of multisampling detection system using a tag insertion primer and an electrochemical DNA chip. Anal Biochem 419: 190-195.

16. Sawata S, Kai E, Ikebukuro K, lida T, Honda T, et al. (1999) Application of peptide nucleic acid to the direct detection of deoxyribonucleic acid amplified by polymerase chain reaction. Biosens Bioelectron 14: 397-404

17. Hatch AC, Ray T, Lintecum K, Youngbull C (2014) Continuous flow real-time PCR device using multi-channel fluorescence excitation and detection. Lab Chip 14: 562-568.

18. Osawa Y, Ikebukuro K, Motoki H, Matsuo T, Horiuchi M, et al. (2008) The simple and rapid detection of specific PCR products from bacterial genomes using Zn finger proteins. Nucleic Acids Res 36: e68.

19. Wolfe SA, Nekludova L, Pabo CO (2000) DNA recognition by Cys2His2 zinc finger proteins. nnu Rev Biophys Biomol Struct 29: 183-212.

20. Pavletich NP, Pabo CO (1991) Zinc finger-DNA recognition: crystal structure of a Zif268-DNA complex at 2.1 A. Science 252: 809-817.

21. Gowetski DB, Kodis EJ, Kahn JD (2013) Rationally designed coiled-coil DNA 
looping peptides control DNA topology. Nucleic Acids Res 41: 8253-8265.

22. Majka J, Jakimowicz D, Zarko-Postawka M, Zakrzewska-Czerwinska J (1997) Glutathione S-transferase fusion proteins as an affinity reagent for rapid isolation of specific sequence directly from genomic DNA. Nucleic Acids Res 25: $2537-2538$

23. Takeuchi A, Sode $K$ (2000) A Salmonella detection system using an engineered DNA binding protein that specifically captured a DNA sequence. Anal Chem 72 2809-2813.

24. Miller J, McLachlan AD, Klug A (1985) Repetitive zinc-binding domains in the protein transcription factor IIIA from Xenopus oocytes. EMBO J 4: 1609-1614.

25. Wolfe SA, Nekludova L, Pabo CO (2000) DNA recognition by Cys2His2 zinc finger proteins. Annu Rev Biophys Biomol Struct 29: 183-212.

26. Nolte RT, Conlin RM, Harrison SC, Brown RS (1998) Differing roles for zinc fingers in DNA recognition: Structure of a six-finger transcription factor IIIA complex. Proc Natl Acad Sci U S A 95: 2938-2943.

27. Stains Cl, Porter JR, Ooi AT, Segal DJ, Ghosh I (2005) DNA sequence-enabled reassembly of the green fluorescent protein. J Am Chem Soc 127: 1078210783.

28. Ooi AT, Stains CI, Ghosh I, Segal DJ (2006) Sequence-enabled reassembly of beta-lactamase (SEER-LAC): A sensitive method for the detection of doublestranded DNA. Biochemistry 45: 3620-3625.

29. Kim MS, Stybayeva G, Lee JY, Revzin A, Segal DJ (2011) A zinc finge protein array for the visual detection of specific DNA sequences for diagnostic applications. Nucleic Acids Res 39: e29.

30. Yoshitake K, Waki S, Ueda H (2008) Dimerization-based homogeneous fluorosensor proteins for the detection of specific dsDNA. Biosens Bioelectron 23: $1266-1271$

31. Osawa Y, Ikebukuro K, Kumagai T, Motoki H, Matsuo T, et al. (2009) Zn fingerbased direct detection system for PCR products of Salmonella spp. and the Influenza A virus. Biotechnol Lett 31: 725-733.

32. Abe K, Kumagai T, Takahashi C, Kezuka A, Murakami Y, et al. (2012) Detection of pathogenic bacteria by using zinc finger protein fused with firefly luciferase. Anal Chem 84: 8028-8032.

33. Ito K, Nishimura W, Maeda M, Gomi K, Inouye S, et al. (2007) Highly sensitive and rapid tandem bioluminescent immunoassay using aequorin labeled Fab fragment and biotinylated firefly luciferase. Anal Chim Acta 588: 245-251.

34. Kricka LJ (2000) Application of bioluminescence and chemiluminescence in biomedical sciences. Methods Enzymol 305: 333-345.

35. Fraga H (2008) Firefly luminescence: A historical perspective and recent developments. Photochem Photobiol Sci 7: 146-158.

36. Ikebukuro K, Kiyohara C, Sode K (2005) Novel electrochemical sensor system for protein using the aptamers in sandwich manner. Biosens Bioelectron 20: 2168-2172.

37. Lee J, Tatsumi A, Abe K, Yoshida W, Sode K, et al. (2014) Electrochemical detection 15 of pathogenic bacteria by using a glucose dehydrogenase fused zinc finger protein. Anal Methods 6: 4991-4994.

38. Chen RZ, Pettersson U, Beard C, Jackson-Grusby L, Jaenisch R (1998) DNA hypo methylation leads to elevated mutation rates. Nature 395: 89-93.

39. Costello JF, Frühwald MC, Smiraglia DJ, Rush LJ, Robertson GP, et al. (2000) Aberrant $\mathrm{CpG}$-island methylation has non-random and tumour-type-specific patterns. Nat Genet 24: 132-138.

40. Hiraoka D, Yoshida W, Abe K, Wakeda H, Hata K, et al. (2012) Development of a method to measure DNA methylation levels by using methyl CpG-binding protein and luciferase-fused zinc finger protein. Anal Chem 84: 8259-8264

41. Gal-Yam EN, Saito Y, Egger G, Jones PA (2008) Cancer epigenetics: Modifications, screening, and therapy. Annu Rev Med 59: 267-280.

42. Yoshida W, Kezuka A, Abe K, Wakeda H, Nakabayashi K, et al. (2013) Detection of histone modification by chromatin immunoprecipitation combined zinc finger luciferase-based bioluminescence resonance energy transfer assay. Anal Chem 85: 6485-6490.

43. Miszczycha SD, Ganet S, Duniere L, Rozand C, Loukiadis E, et al. (2012) Novel real-time PCR method to detect Escherichia coli O157:H7 in raw milk cheese and raw ground meat. J Food Prot 75: 1373-1381.
44. Cheng CY, Chi JR, Lin SR, Chou CC, Huang CC (2009) Rapid quantification of Salmonella typhimurium inoculated to meat products by real-time PCR. Acta Vet Hung 57: 25-38.

45. Yang N, Qi H, Wong MM, Wu RS, Kong RY (2012) Prevalence and diversity of norovirus genogroups I and II in Hong Kong marine waters and detection by real-time PCR. Mar Pollut Bull 64: 164-168.

46. McQuaig SM, Scott TM, Lukasik JO, Paul JH, Harwood VJ (2009) Quantification of human polyomaviruses JC Virus and BK Virus by TaqMan quantitative PCR and comparison to other water quality indicators in water and fecal samples. Appl Environ Microbiol 75: 3379-3388.

47. Furutani S, Naruishi N, Saito M, Tamiya E, Fuchiwaki Y, et al. (2014) Rapid and highly sensitive detection by a real-time polymerase chain reaction using a chip coated with its reagents. Anal Sci 30: 569-574

48. Nixon G, Garson JA, Grant P, Nastouli E, Foy CA, et al. (2014) Comparative study of sensitivity, linearity, and resistance to inhibition of digital and non-digital polymerase chain reaction and loop mediated isothermal amplification assays for quantification of human cytomegalovirus. Anal Chem 86: 4387-4394.

49. Ryan RP, Vorhölter FJ, Potnis N, Jones JB, Van Sluys MA, et al. (2011) Pathogenomics of Xanthomonas: Understanding bacterium-plant interactions. Nat Rev Microbiol 9: 344-355.

50. Rossier O, Wengelnik K, Hahn K, Bonas U (1999) The Xanthomonas Hrp type III system secretes proteins from plant and mammalian bacterial pathogens. Proc Natl Acad Sci U S A 96: 9368-9373.

51. Jankele R, Svoboda P (2014) TAL effectors: Tools for DNA targeting. Brief Funct Genomics 13: 409-419.

52. Deng D, Yan C, Pan X, Mahfouz M, Wang J, et al. (2012) Structural basis for sequence-specific recognition of DNA by TAL effectors. Science 335: 720-723.

53. Fine EJ, Cradick TJ, Zhao CL, Lin Y, Bao G (2014) An online bioinformatics tool predicts zinc finger and TALE nuclease off-target cleavage. Nucleic Acids Res 42: e42.

54. Mak AN, Bradley P, Cernadas RA, Bogdanove AJ, Stoddard BL (2012) The crystal structure of TAL effector PthXo1 bound to its DNA target. Science 335 716-719.

55. Meckler JF, Bhakta MS, Kim MS, Ovadia R, Habrian CH, et al. (2013) Quantitative analysis of TALE-DNA interactions suggests polarity effects. Nucleic Acids Res 41: 4118-4128.

56. Miller JC, Tan S, Qiao G, Barlow KA, Wang J, et al. (2011) A TALE nuclease architecture for efficient genome editing. Nat Biotechnol 29: 143-148.

57. Anthony K, More A, Zhang X (2014) Activation of silenced cytokine gene promoters by the synergistic effect of TBP-TALE and VP64-TALE activators. PLoS One 9: e95790.

58. Joung JK, Sander JD (2013) TALENs: A widely applicable technology for targeted genome editing. Nat Rev Mol Cell Biol 14: 49-55.

59. Urnov FD, Rebar EJ, Holmes MC, Zhang HS, Gregory PD (2010) Genome editing with engineered zinc finger nucleases. Nat Rev Genet 11: 636-646.

60. Li T, Huang S, Jiang WZ, Wright D, Spalding MH, et al. (2011) TAL nucleases (TALNs): Hybrid proteins composed of TAL effectors and FokI DNA-cleavage domain. Nucleic Acids Res 39: 359-372.

61. Watanabe T, Ochiai H, Sakuma T, Horch HW, Hamaguchi N, et al. (2012) Nontransgenic genome modifications in a hemimetabolous insect using zinc-finger and TAL effector nucleases. Nat Commun 3: 1017

62. Wood AJ, Lo TW, Zeitler B, Pickle CS, Ralston EJ, et al. (2011) Targeted genome editing across species using ZFNs and TALENs. Science 333: 307.

63. Tesson L, Usal C, Ménoret S, Leung E, Niles BJ, et al. (2011) Knockout rats generated by embryo microinjection of TALENs. Nat Biotechnol 29: 695-696.

64. Segal DJ, Beerli RR, Blancafort P, Dreier B, Effertz K, et al. (2003) Evaluation of a modular strategy for the construction of novel polydactyl zinc finger DNAbinding proteins. Biochemistry 42: 2137-2148.

65. Maeder ML, Thibodeau-Beganny S, Osiak A, Wright DA, Anthony RM, et al. (2008) Rapid "open-source" engineering of customized zinc-finger nucleases for highly efficient gene modification. Mol Cell 31: 294-301.

66. Sander JD, Dahlborg EJ, Goodwin MJ, Cade L, Zhang F, et al. (2011) Selection- 
Citation: Kumagai T, Abe K, Yoshida W, Ikebukuro K (2015) DNA Detection Technology Using Zinc Finger Protein. J Microb Biochem Technol 7: 278281. doi:10.4172/1948-5948.1000225

free zinc-finger-nuclease engineering by context-dependent assembly (CoDA). Nat Methods 8: 67-69.

67. Moore M, Klug A, Choo Y (2001) Improved DNA binding specificity from polyzinc finger peptides by using strings of two-finger units. Proc Natl Acad Sci U S A 98: 1437-1441.

68. Rebar EJ, Pabo CO (1994) Zinc finger phage: Affinity selection of fingers with new DNA-binding specificities. Science 263: 671-673.

69. Bae KH, Kwon YD, Shin HC, Hwang MS, Ryu EH, et al. (2003) Human zinc fingers as building blocks in the construction of artificial transcription factors. Nat Biotechnol 21: 275-280.

70. Morton J, Davis MW, Jorgensen EM, Carroll D (2006) Induction and repair of zinc-finger nuclease-targeted double-strand breaks in Caenorhabditis elegans somatic cells. Proc Natl Acad Sci U S A 103: 16370-16375.

71. Bibikova M, Golic M, Golic KG, Carroll D (2002) Targeted chromosomal cleavage and mutagenesis in Drosophila using zinc-finger nucleases. Genetics 161: 1169-1175.

72. Cradick TJ, Keck K, Bradshaw S, Jamieson AC, McCaffrey AP (2010) Zincfinger nucleases as a novel therapeutic strategy for targeting hepatitis $B$ virus DNAs. Mol Ther 18: 947-954.

73. Zhu C, Gupta A, Hall VL, Rayla AL, Christensen RG, et al. (2013) Using defined finger-finger interfaces as units of assembly for constructing zinc-finger nucleases. Nucleic Acids Res 41: 2455-2465.

74. Händel EM, Cathomen T (2011) Zinc-finger nuclease based genome surgery: it's all about specificity. Curr Gene Ther 11: 28-37.

75. Greisman HA, Pabo CO (1997) A general strategy for selecting high-affinity zinc finger proteins for diverse DNA target sites. Science 275: 657-661.

76. Meng X, Noyes MB, Zhu LJ, Lawson ND, Wolfe SA (2008) Targeted gene inactivation in zebra fish using engineered zinc-finger nucleases. Nat Biotechnol 26: 695-701.

77. Kim S, Kim JS (2011) Targeted genome engineering via zinc finger nucleases. Plant Biotechnol Rep 5: 9-17.

78. Gupta A, Meng X, Zhu LJ, Lawson ND, Wolfe SA (2011) Zinc finger proteindependent and -independent contributions to the in vivo off-target activity of zinc finger nucleases. Nucleic Acids Res 39: 381-392.

79. Persikov AV, Osada R, Singh M (2009) Predicting DNA recognition by Cys2His2 zinc finger proteins. Bioinformatics 25: 22-29.

80. Cho SY, Chung M, Park M, Park S, Lee YS (2008) ZIFIBI: Prediction of DNA binding sites for zinc finger proteins. Biochem Biophys Res Commun 369: 845848 .

81. Sander JD, Zaback P, Joung JK, Voytas DF, Dobbs D (2007) Zinc Finger Targeter (ZiFiT): An engineered zinc finger/target site design tool. Nucleic Acids Res 35: W599-605.

82. Jayakanthan M, Muthukumaran J, Chandrasekar S, Chawla K, Punetha A, et al. (2009) ZifBASE: A database of zinc finger proteins and associated resources. BMC Genomics 10: 421.

83. Molparia B, Goyal K, Sarkar A, Kumar S, Sundar D (2010) ZiF-Predict: A web tool for predicting DNA-binding specificity in $\mathrm{C} 2 \mathrm{H} 2$ zinc finger proteins Genomics Proteomics Bioinformatics 8: 122-126.

84. Mandell JG, Barbas CF 3rd (2006) Zinc Finger Tools: Custom DNA-binding domains for transcription factors and nucleases. Nucleic Acids Res 34: W516523.

85. Fu F, Voytas DF (2013) Zinc Finger Database (ZiFDB) v2.0: A comprehensive database of Câ, Hâ, zinc fingers and engineered zinc finger arrays. Nucleic Acids Res 41: D452-455.

86. Workman CT, Yin Y, Corcoran DL, Ideker T, Stormo GD, et al. (2005) enoLOGOS: A versatile web tool for energy normalized sequence logos. Nucleic Acids Res 33: W389-392.

87. Persikov AV, Singh M (2014) De novo prediction of DNA-binding specificities for Cys2His2 zinc finger proteins. Nucleic Acids Res 42: 97-108.

88. Roy S, Dutta S, Khanna K, Singla S, Sundar D (2012) Prediction of DNAbinding specificity in zinc finger proteins. J Biosci 37: 483-491.

89. Maeder ML, Angstman JF, Richardson ME, Linder SJ, Cascio VM, et al. (2013) Targeted DNA demethylation and activation of endogenous genes using programmable TALE-TET1 fusion proteins. Nat Biotechnol 31: 1137-1142.

90. Zhu C, Gupta A, Hall VL, Rayla AL, Christensen RG, et al. (2013) Using defined finger-finger interfaces as units of assembly for constructing zinc-finger nucleases. Nucleic Acids Res 41: 2455-2465.

91. Gupta A, Christensen RG, Bell HA, Goodwin M, Patel RY, et al. (2014) An improved predictive recognition model for Cys(2)-His(2) zinc finger proteins. Nucleic Acids Res 42: 4800-4812.

92. Persikov AV, Rowland EF, Oakes BL, Singh M, Noyes MB (2014) Deep sequencing of large library selections allows computational discovery of diverse sets of zinc fingers that bind common targets. Nucleic Acids Res 42: 1497-1508.

93. Persikov AV, Singh M (2014) De novo prediction of DNA-binding specificities for Cys2His2 zinc finger proteins. Nucleic Acids Res 42: 97-108.

94. Pearson $\mathrm{H}$ (2008) Protein engineering: The fate of fingers. Nature 455: 160-164.

95. Segal DJ, Dreier B, Beerli RR, Barbas CF (1999) Toward controlling gene expression at will: Selection and design of zinc finger domains recognizing each of the 5'-GNN-3' DNA target sequences. Proc Natl Acad Sci U S A 96: 2758-2763.

96. Dreier B, Beerli RR, Segal DJ, Flippin JD, Barbas CF 3rd (2001) Development of zinc finger domains for recognition of the 5'-ANN-3' family of DNA sequences and their use in the construction of artificial transcription factors. J Biol Chem 276: 29466-29478.

97. Liu Q, Xia Z, Zhong X, Case CC (2002) Validated zinc finger protein designs for all 16 GNN DNA triplet targets. J Biol Chem 277: 3850-3856.

98. Bae KH, Kwon YD, Shin HC, Hwang MS, Ryu EH, et al. (2003) Human zinc fingers as building blocks in the construction of artificial transcription factors. Nat Biotechnol 21: 275-280.

99. Dreier B, Fuller RP, Segal DJ, Lund CV, Blancafort P, et al. (2005) Development of zinc finger domains for recognition of the $5^{\prime}-\mathrm{CNN}-3$ ' family DNA sequences and their use in the construction of artificial transcription factors. J Biol Chem 280: 35588-35597. 